\title{
Perfil terapêutico da hipertensão na Rede Médicos Sentinela - 12 anos depois
}

Diana Souto, ${ }^{1}$ José Augusto Simões, ${ }^{2}$ Carla Torre, ${ }^{3}$ Zilda Mendes, ${ }^{4}$ Isabel Marinho Falcão, ${ }^{5}$ Fernando Ferreira, ${ }^{6}$ Ana da Costa Miranda, Carlos Matias Dias ${ }^{8}$

\section{RESUMO}

Objectivos: Determinar o perfil terapêutico da hipertensão arterial na Rede de Médicos Sentinela e comparar os resultados obtidos, 12 anos após a realização do primeiro estudo.

Tipo de estudo: Estudo transversal.

Local: Rede de Médicos Sentinela de Portugal.

População: Doentes hipertensos com mais de 18 anos das listas dos médicos sentinela que se deslocaram à consulta entre 7 de Junho de 2010 e 31 de Dezembro de 2010.

Métodos: Os médicos sentinela participantes recolheram informação dos doentes hipertensos, informação essa que contemplou a caracterização sociodemográfica, a caracterização da patologia (data de diagnóstico, forma de hipertensão, danos em órgão-alvo e morbilidade associada) e a caracterização da terapêutica instituída.

Resultados: Foram incluídos 616 hipertensos no estudo. Comparativamente ao primeiro estudo (1998), verificou-se um ligeiro aumento da idade média dos participantes (62,0 anos em 1998 vs 64,3 anos em 2010), mantendo-se o predomínio do sexo feminino. A duração média da hipertensão desde o momento do diagnóstico aumentou significativamente $(p<0,0001)$. Aproximadamente metade dos hipertensos apresentou danos em órgãos-alvos em ambos os momentos, sendo a doença cardíaca o dano mais frequente. As patologias associadas mais frequentes foram a dislipidemia, a hiperuricemia e a diabetes. De 1998 para 2010, a proporção de doentes em monoterapia diminuiu (47,6\% vs 30,3\%), sendo os inibidores da enzima da conversão da angiotensina a classe terapêutica mais frequentemente prescrita em monoterapia nos dois momentos. Em 1998, o padrão de tratamento mais frequentemente prescrito foram inibidores da enzima da conversão da angiotensina em monoterapia (24,3\%) e em 2010 foi a associação de antagonistas dos receptores da angiotensina + diuréticos tiazídicos (15,4\%).

Conclusões: Os fármacos que actuam no eixo renina-angiotensina-aldosterona foram, neste estudo, os mais frequentemente prescritos em ambos os momentos, tanto em monoterapia como em politerapia. $O$ número de anti-hipertensores prescritos diferiu entre os dois momentos de acordo com a existência e tipo de comorbilidade.

Palavras-chave: Hipertensão; Perfil de Prescrição Médica; Uso Terapêutico; Médicos de Cuidados de Saúde Primários.

\section{INTRODUÇÃO}

A hipertensão arterial (HTA) é um importante factor de risco no desenvolvimento de doenças cardiovasculares, como o enfarte do miocárdio e o acidente vascular cerebral (AVC), sendo um dos mais importantes problemas de saúde em Portugal, causando excesso de morbilidade e mortalidade. ${ }^{1}$

Estima-se que mais de três milhões de adultos portugueses sofram de HTA. ${ }^{2}$ No estudo de Mário Espiga de Macedo e colaboradores, ${ }^{2}$ apenas $11,2 \%$ dos hipertensos tinham a pressão arterial (PA) controlada e ape- nas $28,9 \%$ dos que relataram tomar a sua medicação, regularmente, tinham a sua PA controlada. A definição de HTA controlada utilizada no estudo citado foi considerar uma pressão arterial sistólica (PAS) < $140 \mathrm{mmHg}$ e uma pressão arterial diastólica (PAD) $<90 \mathrm{mmHg}$, o que se encontra de acordo com as recomendações europeias $^{3} \mathrm{e}$ com a norma emitida pela Direcção-Geral da Saúde (DGS) em 2011.4

O controlo da HTA é imperativo, não só para reduzir o risco de enfarte do miocárdio, AVC e doença renal crónica mas também a incidência de fibrilhação auri- 
cular, insuficiência cardíaca congestiva e disfunção cognitiva. Vários agentes anti-hipertensivos provaram ser eficazes, ${ }^{5}$ tendo sido demonstrado que uma redução de $10 \mathrm{mmHg}$ na PAS e uma redução de $5 \mathrm{mmHg}$ na PAD diminui significativamente a incidência de enfarte do miocárdio, AVC, insuficiência cardíaca congestiva e a mortalidade global. ${ }^{6}$ No entanto, a reduzida proporção de hipertensos controlados sugere a necessidade de abordagens terapêuticas mais agressivas, bem como de melhoria nos sistemas de prestação de cuidados de saúde. ${ }^{1,2,6}$

Além das complicações associadas, a HTA não controlada representa um importante impacto económico, decorrente da incapacidade por ela provocada, bem como dos elevados custos associados ao seu tratamento. Em Portugal, em 2012, os anti-hipertensores (aHT) foram o subgrupo terapêutico com maior encargo financeiro para o Serviço Nacional de Saúde. ${ }^{7}$

A elevada prevalência da HTA na população portuguesa, o seu insuficiente controlo e o decorrente elevado impacto económico, fazem da HTA uma das áreas prioritárias de intervenção em saúde. A DGS estabeleceu o Programa Nacional para as Doenças Cérebro-Cardiovasculares como um dos programas de saúde prioritários em Portugal. Além da promoção da prevenção cardiovascular e da prestação racional de cuidados diagnósticos e terapêuticos, este programa tem também como objectivo a promoção das boas práticas clínicas e terapêuticas através da adopção de recomendações clínicas nacionais ou internacionais.

Para o planeamento e implementação das estratégias destinadas a melhorar o tratamento da HTA e para a monitorização dos indicadores estabelecidos pela DGS é necessária a caracterização dos padrões de tratamento e da sua evolução ao longo dos anos.

Desta forma, a Rede de Médicos Sentinela (MS) decidiu repetir em 2010, 12 anos depois do estudo transversal realizado em 1998, o estudo do perfil terapêuti-

\footnotetext{
${ }^{1}$ Farmacêutica, Centro de Estudos e Avaliação em Saúde

${ }^{2}$ Médico Sentinela, USF Marquês de Marialva, Cantanhede

${ }^{3}$ Farmacêutica e Epidemiologista, Centro de Estudos e Avaliação em Saúde

${ }^{4}$ Estatística, Centro de Estudos e Avaliação em Saúde

${ }^{5}$ Médica de Medicina Geral e Familiar, Direcção-Geral da Saúde

${ }^{6}$ Médico Sentinela, USF de Sto. André de Canidelo, Vila Nova de Gaia

${ }^{7}$ Médica Epidemiologista, Centro de Estudos e Avaliação em Saúde

${ }^{8}$ Médico de Saúde Pública, Departamento de Epidemiologia do Instituto Nacional de Saúde Doutor Ricardo Jorge e Escola Nacional de Saúde Pública Universidade Nova de Lisboa
}

co da HTA na Rede. ${ }^{8}$

Este estudo teve como principais objectivos determinar o perfil terapêutico da HTA na Rede de MS, de acordo com as variáveis demográficas (idade, sexo), forma de HTA e comorbilidades e comparar os resultados obtidos, 12 anos após a realização do primeiro estudo.

\section{MÉTODOS}

Realizou-se um estudo transversal, tendo-se recorrido à Rede MS, que consiste numa rede de médicos de família, cuja actividade profissional se desenvolve em Centros de Saúde do Serviço Nacional de Saúde e que, voluntariamente, notificam casos ou episódios de doença e de outras situações relacionadas com a saúde dos indivíduos inscritos nas respectivas listas de utentes. No ano 2010 estavam inscritos na Rede MS 152 médicos de família, distribuídos nos 18 distritos do Continente e nas Regiões Autónomas dos Açores e da Madeira. Em Abril de 2010 foi enviado um convite a todos os médicos da Rede para colaborarem no estudo, tendo aceite participar 35 médicos.

A selecção de doentes foi efectuada sequencialmente, de acordo com o plano de consultas de cada médico e com base num plano de amostragem por cotas. Tendo em conta o número de médicos que aceitou participar, foi pedido a cada médico que recolhesse entre 27 e 31 casos, seguindo a distribuição por cotas descrita no Quadro I (de acordo com a distribuição por sexo e classe etária dos doentes hipertensos constante no Inquérito Nacional de Saúde de 2005/2006).

A recolha dos dados decorreu entre 7 de Junho de 2010 e 31 de Dezembro de 2010, tendo sido incluídos os indivíduos hipertensos, com idade igual ou superior a 18 anos, que consultaram o médico de família no período considerado, independentemente do diagnóstico. As mulheres grávidas foram excluídas.

Para o cálculo da dimensão da amostra fixou-se um erro absoluto de $3 \%$ e um intervalo de confiança de $95 \%$ para o cálculo de proporções, tendo-se obtido uma dimensão de amostra de 1.067 doentes hipertensos.

O questionário preenchido pelo médico de família compreendeu informação sobre a caracterização do doente (sexo, idade e etnia), a caracterização da patologia (data aproximada do diagnóstico, forma da HTA, danos em órgãos-alvo e morbilidade associada) e a te- 


\begin{tabular}{|c|c|c|}
\hline \multicolumn{3}{|c|}{$\begin{array}{l}\text { QUADRO I. Distribuição da amostra a recrutar segundo } \\
\text { o sexo e idade. }\end{array}$} \\
\hline Grupo etário & N. ${ }^{\circ}$ Homens & N. ${ }^{\circ}$ Mulheres \\
\hline 18 a 24 anos & 0 ou 1 & 0 ou 1 \\
\hline 25 a 34 anos & 0 ou 1 & 0 ou 1 \\
\hline 35 a 44 anos & 1 & 1 \\
\hline 45 a 54 anos & 2 & 3 \\
\hline 55 a 64 anos & 3 & 4 \\
\hline 65 a 74 anos & 3 & 4 \\
\hline 75 anos ou mais & 2 & 4 \\
\hline
\end{tabular}

rapêutica farmacológica.

No que respeita à caracterização da patologia, a variável forma de HTA foi classificada em três estratos, em função dos valores de PAS e PAD no momento do diagnóstico: grau 1 ou ligeira (PAS 140-159 mmHg e PAD 90$-99 \mathrm{mmHg}$ ), grau 2 ou moderada (PAS 160-179 mmHg e PAD 100-109 $\mathrm{mmHg}$ ) e grau 3 ou severa (PAS $\geq 180$ $\mathrm{mmHg}$ e $\mathrm{PAD} \geq 110 \mathrm{mmHg}$ ).

Os danos em órgão-alvo (doença cardíaca, insuficiência renal, doença cérebro-vascular, retinopatia e doença arterial periférica) e morbilidade(s) associada(s) (diabetes, asma, doença pulmonar obstrutiva crónica, disfunção eréctil, glaucoma, insuficiência hepática, dislipidemia, hiperuricemia e perturbações da condução aurículo-ventricular) foram definidos de acordo com o critério clínico de cada médico participante, recorrendo, se necessário, ao processo clínico do doente.

Para análise comparativa e conforme descrito, anteriormente, utilizaram-se os resultados do estudo realizado em $1998 .^{8}$

\section{Análise Estatística}

Os dados recolhidos foram introduzidos numa base de dados expressamente construída para o efeito, em Microsoft Access. Foi realizada uma validação por amostragem dos dados recolhidos e introduzidos na base de dados, comparando os questionários recepcionados em papel com o registo informático. A análise estatística foi realizada no programa informático SAS GUIDE versão 4.0.

A análise consistiu na descrição de todas as variáveis em estudo, através do cálculo de frequências absolutas, frequências relativas, medidas de localização e medidas de dispersão. Para as análises relacionadas com o perfil terapêutico foram calculados os intervalos de confiança a 95\% com aproximação à normal sem correcção de continuidade, para as proporções encontradas. Para a comparação de alguns resultados, segundo as características dos doentes, utilizou-se o teste de Qui-quadrado, ou o teste exacto de Fisher, em dados categorizados, e o teste não paramétrico de Mann-Whitneye Wilcoxon para dados contínuos.

A análise comparativa dos resultados obtidos em 1998 e os resultados obtidos em 2010 foi efectuada recorrendo ao teste de Qui-quadrado ou ao teste de comparação de proporções. No caso das variáveis idade e tempo de HTA foi utilizado o teste F de Snedecor para testar a hipótese de igualdade entre as variâncias e posteriormente o teste $t$-student para comparação de médias com aproximação de Welch. Foi utilizado um nível de significância $\alpha=0,05$. Os valores omissos foram excluídos da análise.

\section{RESULTADOS}

Em 2010, foram incluídos no estudo 616 doentes hipertensos. O Quadro II apresenta as características gerais da amostra nos dois momentos em estudo. Relativamente às variáveis sociodemográficas, observaram-se diferenças significativas ao nível da idade e do sexo entre os dois momentos, verificando-se um aumento da idade média dos participantes (IC 95\%: 63,2-65,4) e um aumento da proporção dos participantes do sexo masculino, de 1998 para 2010. Em ambos os momentos, apenas $1 \%$ dos doentes não era de etnia caucasiana, pelo que se considerou que essa característica não era relevante para a análise.

Relativamente à duração da HTA, de 1998 para 2010, diminuiu a proporção de doentes com duração da HTA superior a 10 anos, sendo esta diferença estatisticamente significativa. Não se verificaram diferenças quanto à duração média da HTA. No que respeita à forma de HTA no momento do diagnóstico, verificou-se um aumento da proporção de doentes com HTA ligeira, a par de uma diminuição da proporção de participantes com HTA moderada. Aproximadamente metade dos hipertensos apresentou danos em órgãos-alvos em ambos os momentos, sendo a doença cardíaca o 
dano mais frequente. As patologias associadas mais frequentes foram, em 1998 e 2010, a dislipidemia, a hiperuricemia e a diabetes. No entanto, a proporção de participantes com dislipidemia e diabetes aumentou e a proporção com hiperuricemia diminuiu (Quadro II).

Entre 1998 e 2010, o padrão de prescrição revelou diferenças relativas à prescrição de aHT em monoterapia ou politerapia e também no que respeita às classes de aHT prescritas. O esquema terapêutico em monoterapia foi prescrito a $47,6 \%$ ( $\mathrm{n}=536$; IC95\%: 44,7\%-50,5\%) dos hipertensos em 1998 e a $30,3 \%$ ( $\mathrm{n}=183$; IC95\%: 26,7\%-34,0\%) em 2010 (Figura 1). Neste esquema, as classes terapêuticas mais frequentemente prescritas foram, em 1998, os inibidores da enzima da conversão da angiotensina (IECA) $(24,3 \%$; IC95\%:20,7\%-27,9\%), seguidos dos bloqueadores dos canais de cálcio (BCC) (8,4\%; IC95\%: 6,1\%-10,7\%) e diuréticos (6,1\%; IC95\%: 4,1\%-8,1\%). Em 2010, o uso de IECA, BCC e diuréticos diminuiu para 10,8\% (IC95\%: 6,3\%-15,3\%), 3,2\% (IC95\%: 0,6\%$-5,7 \%$ ) e $2,2 \%$ (IC95\%: 0,1\%-4,3\%), respetivamente.

A classe dos IECA representou, em ambos os momentos, a classe mais frequentemente prescrita em monoterapia, tendo diminuído de $24,3 \%$ para $10,3 \%$ em 2010.

A prescrição de antagonistas dos receptores da angiotensina (ARA) aumentou significativamente de 3,2\% (IC95\%: 1,7\%-4,7\%) em 1998 para 9,8\% (IC95\%: 5,5\%-14,1\%) em 2010. A prescrição de bloqueadores beta (BB) não diferiu significativamente entre os dois momentos, variando de 5,2\% (IC95\%: 3,3\%-7,1\%)

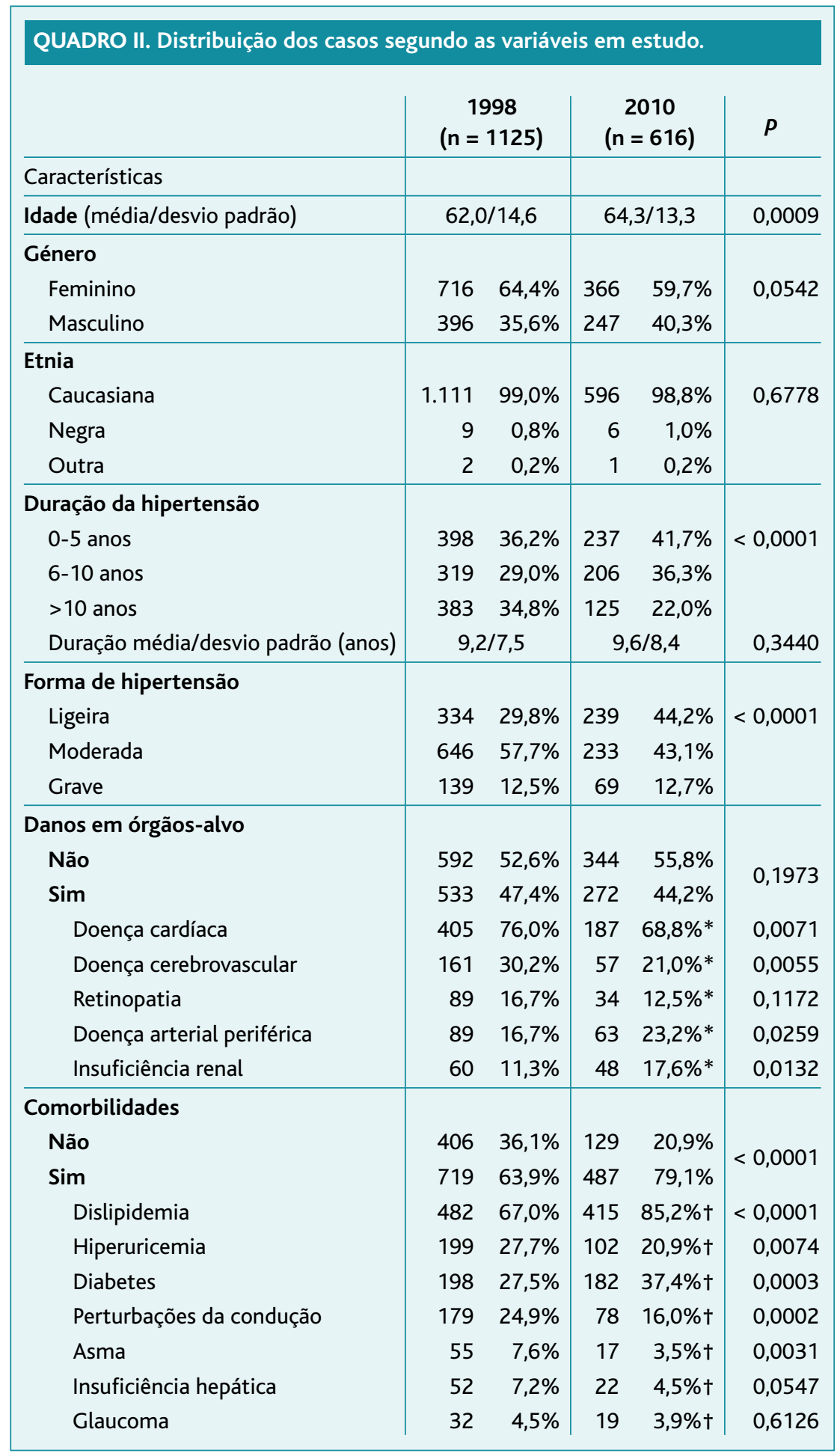

*\% sobre o total de casos com pelo menos um dano em órgão-alvo †\% sobre o total de casos com pelo menos uma comorbilidade 


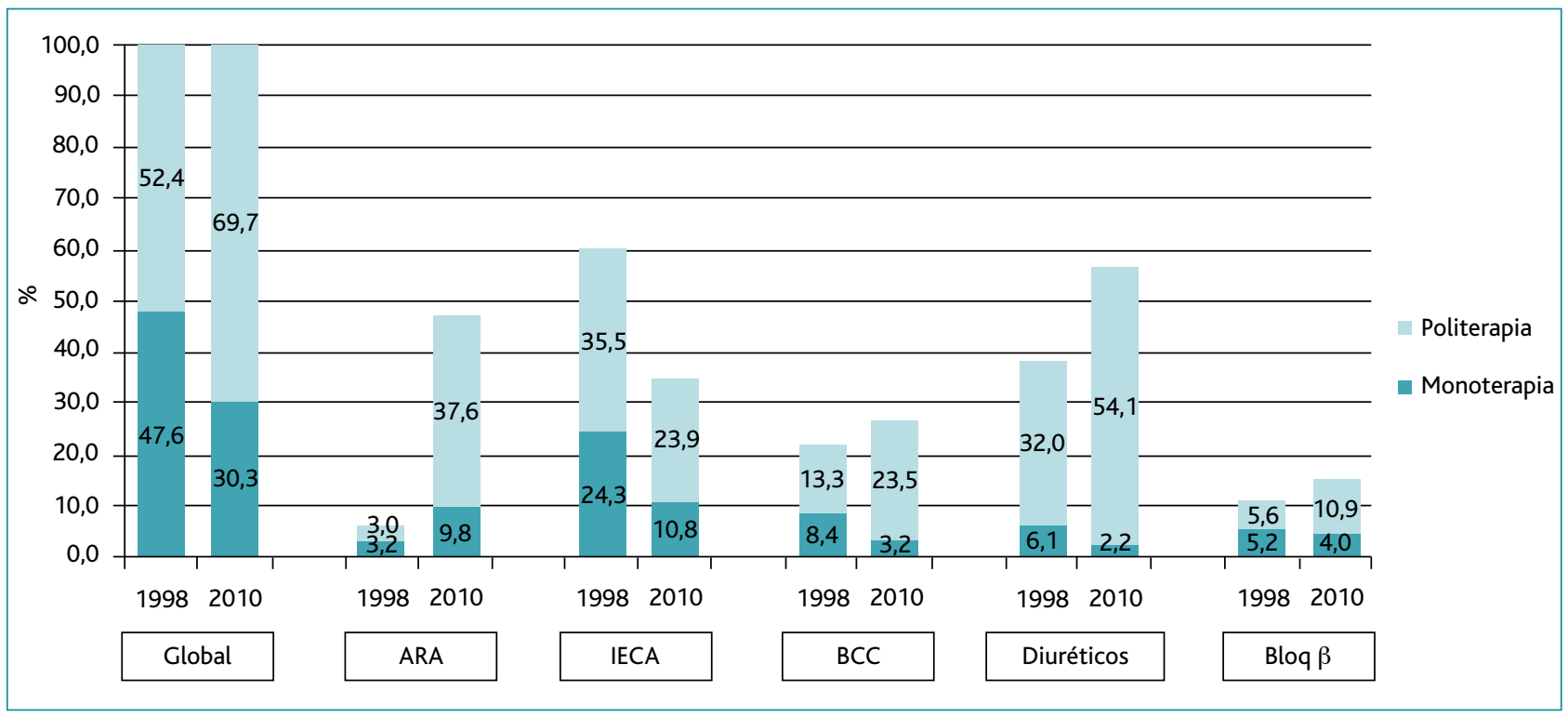

Figura 1. Anti-hipertensores prescritos em monoterapia vs politerapia: prescrição de fármacos anti-hipertensores por grupo terapêutico. ARA: antagonistas dos receptores da angiotensina; IECA: inibidores da enzima de conversão da angiotensina; BCC: bloqueadores dos canais de cálcio; Bloq $\beta$ : bloqueadores beta.

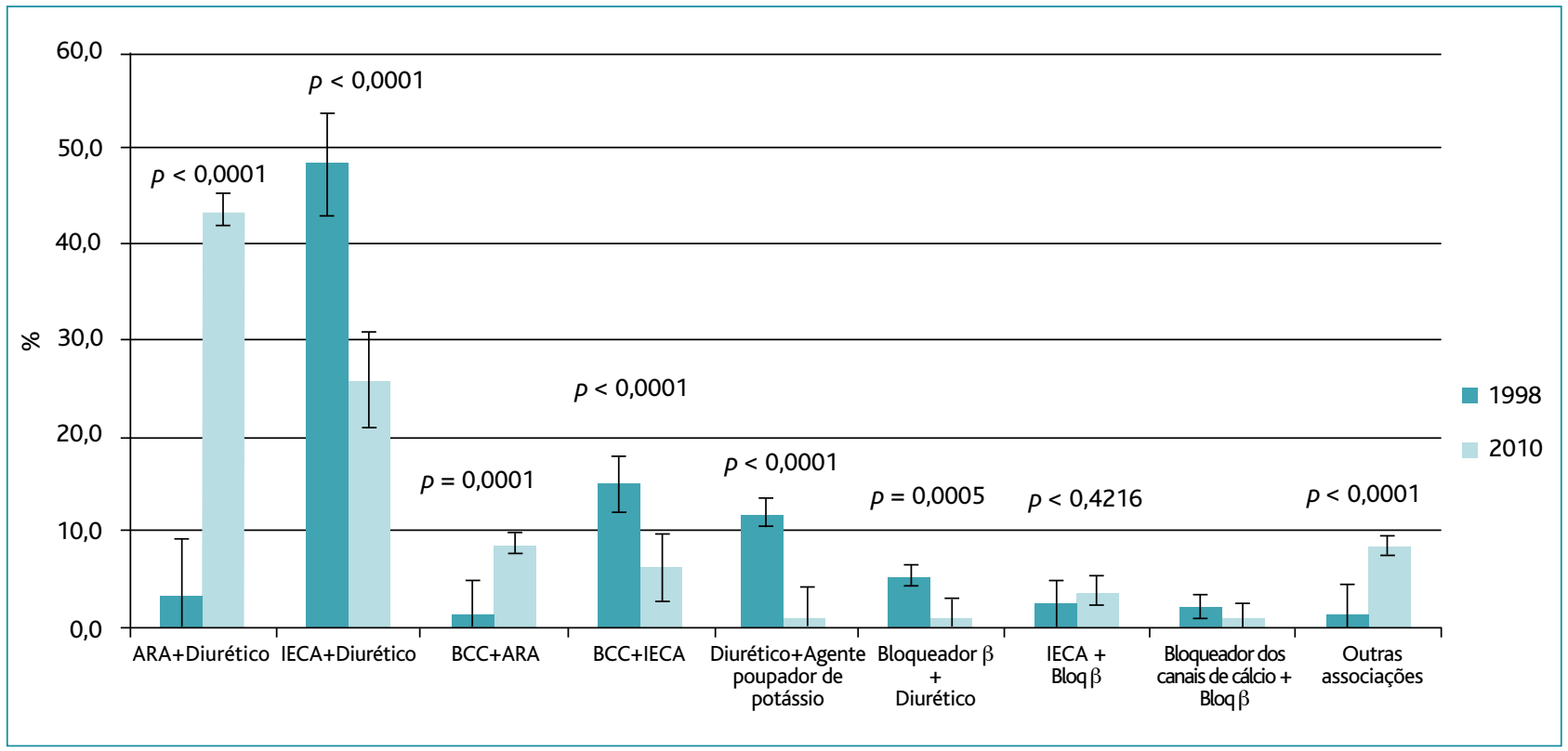

Figura 2. Padrões de tratamento anti-hipertensor: utilização de 2 classes terapêuticas de anti-hipertensores.

ARA: antagonistas dos receptores da angiotensina; IECA: inibidores da enzima de conversão da angiotensina; BCC: bloqueadores dos canais de cálcio; Bloq $\beta$ : bloqueadores beta.

para 4,0\% (IC95\%: 1,1\%-6,8\%).

Entre 1998 e 2010 o número médio de aHT prescritos manteve-se semelhante (1,4 vs 1,5 medicamentos por doente).
A combinação de 2 classes de aHT continuou a ser o modo mais comum de politerapia entre 1998 (36,2\%; IC95\%: 33,4\%-39,0\%) e 2010 (42,8\%; IC95\%: 38,8\%$-46,7 \%)$. De entre as possíveis combinações entre as 


\begin{tabular}{|c|c|c|c|c|c|c|c|c|}
\hline & \multicolumn{2}{|c|}{$\begin{array}{c}\text { Sem } \\
\text { Comorbilidades } \\
P=0,0032^{*}\end{array}$} & \multicolumn{2}{|c|}{$\begin{array}{c}\text { Diabetes } \\
P=0,2764^{*}\end{array}$} & \multicolumn{2}{|c|}{$\begin{array}{l}\text { Dislipidemia } \\
P=0,0233^{*}\end{array}$} & \multicolumn{2}{|c|}{$\begin{array}{c}\text { Hiperuricemia } \\
p=0,8353^{*}\end{array}$} \\
\hline & 1998 & 2010 & 1998 & 2010 & 1998 & 2010 & 1998 & 2010 \\
\hline Monoterapia & $\begin{array}{r}209 \\
(52,4 \%)\end{array}$ & $\begin{array}{r}40 \\
(34,2 \%)\end{array}$ & $\begin{array}{r}26 \\
(48,1 \%)\end{array}$ & $\begin{array}{r}7 \\
(35,0 \%)\end{array}$ & $\begin{array}{r}98 \\
(53,0 \%)\end{array}$ & $\begin{array}{r}61 \\
(37,7 \%)\end{array}$ & $\begin{array}{r}15 \\
(42,8 \%)\end{array}$ & $\begin{array}{r}6 \\
(46,2 \%)\end{array}$ \\
\hline 2 fármacos & $\begin{array}{r}145 \\
(36,3 \%)\end{array}$ & $\begin{array}{r}57 \\
(48,7 \%)\end{array}$ & $\begin{array}{r}17 \\
(31,5 \%)\end{array}$ & $\begin{array}{r}6 \\
(30,0 \%)\end{array}$ & $\begin{array}{r}63 \\
(34,0 \%)\end{array}$ & $\begin{array}{r}65 \\
(40,1 \%)\end{array}$ & $\begin{array}{r}11 \\
(31,4 \%)\end{array}$ & $\begin{array}{r}3 \\
23,1 \%)\end{array}$ \\
\hline 3 fármacos & $\begin{array}{r}39 \\
(9,8 \%) \\
\end{array}$ & $\begin{array}{r}15 \\
(12,8 \%) \\
\end{array}$ & $\begin{array}{r}11 \\
(20,4 \%) \\
\end{array}$ & $\begin{array}{r}6 \\
(30,0 \%) \\
\end{array}$ & $\begin{array}{r}20 \\
(10,8 \%) \\
\end{array}$ & $\begin{array}{r}29 \\
(17,9 \%) \\
\end{array}$ & $\begin{array}{r}8 \\
(22.9 \%) \\
\end{array}$ & $\begin{array}{r}4 \\
30,8 \%) \\
\end{array}$ \\
\hline 4 fármacos & $\begin{array}{r}6 \\
(1,5 \%)\end{array}$ & $\begin{array}{r}5 \\
(4,3 \%)\end{array}$ & $\begin{array}{r}0 \\
(0,0 \%)\end{array}$ & $\begin{array}{r}0 \\
(0,0 \%)\end{array}$ & $\begin{array}{r}4 \\
(2,2 \%)\end{array}$ & $\begin{array}{r}6 \\
(3,7 \%)\end{array}$ & $\begin{array}{r}1 \\
(2,9 \%)\end{array}$ & $\begin{array}{r}0 \\
(0,0 \%)\end{array}$ \\
\hline Total & $\begin{array}{r}399 \\
(100 \%)\end{array}$ & $\begin{array}{r}117 \\
(100 \%)\end{array}$ & $\begin{array}{r}54 \\
(100 \%)\end{array}$ & $\begin{array}{r}20 \\
(100 \%)\end{array}$ & $\begin{array}{r}185 \\
(100 \%)\end{array}$ & $\begin{array}{r}162 \\
(100 \%)\end{array}$ & $\begin{array}{r}35 \\
(100 \%)\end{array}$ & $\begin{array}{r}13 \\
(100 \%)\end{array}$ \\
\hline
\end{tabular}

* $p$ value para as diferenças entre o número de anti-hipertensores prescritos em 1998 e 2010, de acordo com a existência e tipo de comorbilidades.

principais classes de medicamentos aHT, verificou-se um aumento significativo para a prescrição de ARA em combinação com um diurético (3,2\%; IC95\%: 1,5\%$-4,9 \%$ vs 43,4\%; IC95\%: 37,4\%-39,5\%) ou um BCC (1,5; IC95\%: 0,3\% 2,7\% vs 8,9\%; IC95\%: 5,4\%-12,4\%) e uma diminuição de associações de IECA em combinações com diurético (48,2\%; IC95\%: 43,4\%-53,0\% vs 26,0\%; IC95\%: 20,6\%-31,3\%) ou BCC (15,0 IC95\%: $11,5 \%-18,5 \%$ vs 6,2\%; IC95\%: 3,3\%-9,1\%) (Figura 2).

A politerapia contendo 3 classes de medicamentos aHT aumentou de 14,0\% (IC95\%: 12,0\%-16,0\%) para 21,2\% (IC95\%: 18,0\%-24,5\%). Em 2010, as três formas mais comuns deste tipo de politerapia foram as combinações que incluíam um diurético: diurético + ARA + BCC (22,7\%; IC95\%: 18,7\%-26,7\%), diurético + ARA + BB (14,1\%; IC95\%: 10,8\%-17,4\%) ou diurético + IECA + BCC (13,3\%; IC95\%: 10,1\%-16,5\%).

Em 2010, os padrões de tratamento mais frequentes foram os ARA + diuréticos tiazídicos (15,4\%; IC95\%: $12,5 \%-18,3 \%)$, seguido de IECA em monoterapia (10,8\%; IC95\%: 6,3\%-15,3\%) e ARA (9,8\%; IC95\%: 5,5\%$14,1 \%)$.

De forma a avaliar o efeito da presença de comorbilidades na terapêutica farmacológica da HTA, compararam-se os esquemas terapêuticos instituídos aos doentes sem qualquer comorbilidade com os esquemas instituídos aos doentes com apenas uma comorbilidade (diabetes, dislipidemia ou hiperuricemia). Foram observadas diferenças significativas em relação ao número de fármacos aHT prescritos na presença de diabetes $(p=0,0472)$, dislipidemia $(p=0,0431)$ e hiperuricemia $(p=0,0463)$ (Quadro III). Comparando o número de aHT prescritos em 1998 com número de aHT prescritos em 2010 em função da existência e tipo de comorbilidades, observaram-se diferenças significativas entre os dois momentos, na ausência de comorbilidades $(p=0,0032)$ e para a dislipidemia $(p=0,0233)$ (Quadro III).

Quanto à distribuição dos grupos terapêuticos mais prescritos de acordo com a existência e tipo de comorbilidade, verificou-se um aumento significativo na prescrição de ARA, isolados ou em associação, tanto nos indivíduos sem comorbilidades, como nos indivíduos com apenas uma comorbilidade (diabetes, dislipidemia ou hiperuricemia) (Quadro IV). Em 2010, a proporção de doentes sob terapêutica com IECA diminuiu para todas as patologias apresentadas, tendo sido a classe mais frequentemente prescrita em 1998, independentemente da presença ou tipo de patologia (Quadro IV). Tendo em consideração que os ARA eram uma classe 


\begin{tabular}{|c|c|c|c|c|c|c|c|c|}
\hline & \multicolumn{2}{|c|}{$\begin{array}{c}\text { Sem } \\
\text { Comorbilidades }\end{array}$} & \multicolumn{2}{|c|}{ Diabetes } & \multicolumn{2}{|c|}{ Dislipidemia } & \multicolumn{2}{|c|}{ Hiperuricemia } \\
\hline & 1998 & 2010 & 1998 & 2010 & 1998 & 2010 & 1998 & 2010 \\
\hline $\begin{array}{l}\text { ARA + Diuréticos } \\
\text { tiazídicos }\end{array}$ & $\begin{array}{r}2 \\
(1,1 \%)\end{array}$ & $\begin{array}{r}21 \\
(41,2 \%)\end{array}$ & $\begin{array}{r}0 \\
(0,0 \%)\end{array}$ & $\begin{array}{r}3 \\
(17,7 \%)\end{array}$ & $\begin{array}{r}2 \\
(2,4 \%)\end{array}$ & $\begin{array}{r}22 \\
(33,9 \%)\end{array}$ & $\begin{array}{r}0 \\
(0,0 \%)\end{array}$ & $\begin{array}{r}2 \\
(14,3 \%)\end{array}$ \\
\hline IECA & $\begin{array}{r}90 \\
(50,6 \%)\end{array}$ & $\begin{array}{r}13 \\
(25,5 \%)\end{array}$ & $\begin{array}{r}18 \\
(75,0 \%)\end{array}$ & $\begin{array}{r}2 \\
(11,8 \%)\end{array}$ & $\begin{array}{r}52 \\
(63,4 \%)\end{array}$ & $\begin{array}{r}23 \\
(35,4 \%)\end{array}$ & $\begin{array}{r}8 \\
(66,7 \%)\end{array}$ & $\begin{array}{r}2 \\
(14,3 \%)\end{array}$ \\
\hline ARA & $\begin{array}{r}13 \\
(7,3 \%)\end{array}$ & $\begin{array}{r}12 \\
(23,5 \%)\end{array}$ & $\begin{array}{r}0 \\
(0,0 \%)\end{array}$ & $\begin{array}{r}2 \\
(11,8 \%)\end{array}$ & $\begin{array}{r}0 \\
(0,0 \%)\end{array}$ & $\begin{array}{r}15 \\
(23,1 \%)\end{array}$ & $\begin{array}{r}0 \\
(0,0 \%)\end{array}$ & $\begin{array}{r}3 \\
(21,4 \%)\end{array}$ \\
\hline $\begin{array}{l}\text { IECA + Diuréticos } \\
\text { tiazídicos }\end{array}$ & $\begin{array}{r}72 \\
(40,4 \%)\end{array}$ & $\begin{array}{r}0 \\
(0,0 \%)\end{array}$ & $\begin{array}{r}5 \\
(20,8 \%)\end{array}$ & $\begin{array}{r}9 \\
(52,9 \%)\end{array}$ & $\begin{array}{r}28 \\
(34,1 \%)\end{array}$ & $\begin{array}{r}0 \\
(0,0 \%)\end{array}$ & $\begin{array}{r}4 \\
(33,3 \%)\end{array}$ & $\begin{array}{r}6 \\
(42,9 \%)\end{array}$ \\
\hline $\begin{array}{l}\mathrm{BCC}+\mathrm{ARA}+ \\
\text { Diuréticos tiazídicos }\end{array}$ & $\begin{array}{r}1 \\
(0,6 \%)\end{array}$ & $\begin{array}{r}5 \\
(9,8 \%)\end{array}$ & $\begin{array}{r}1 \\
(4,2 \%)\end{array}$ & $\begin{array}{r}1 \\
(5,9 \%)\end{array}$ & $\begin{array}{r}0 \\
(0,0 \%)\end{array}$ & $\begin{array}{r}5 \\
(7,7 \%)\end{array}$ & $\begin{array}{r}0 \\
(0,0 \%)\end{array}$ & $\begin{array}{r}1 \\
(7,1 \%)\end{array}$ \\
\hline Total & $\begin{array}{r}178 \\
(100 \%)\end{array}$ & $\begin{array}{r}51 \\
(100 \%)\end{array}$ & $\begin{array}{r}24 \\
(100 \%)\end{array}$ & $\begin{array}{r}17 \\
(100 \%)\end{array}$ & $\begin{array}{r}82 \\
(100 \%)\end{array}$ & $\begin{array}{r}65 \\
(100 \%)\end{array}$ & $\begin{array}{r}12 \\
(100 \%)\end{array}$ & $\begin{array}{r}14 \\
(100 \%)\end{array}$ \\
\hline
\end{tabular}

relativamente recente em 1998, a frequência da sua utilização no referido ano era muito inferior comparando com 2010. Não foi possível aplicar o teste estatístico para comparar os grupos terapêuticos prescritos em 1998 e em 2010 em função da presença e tipo de comorbilidades devido ao reduzido número de casos após estratificação da amostra.

Para este estudo foram ainda analisadas algumas características dos MS, nomeadamente a idade, o sexo e os anos de prática clínica que se considerou poderem, eventualmente, influenciar a decisão no que respeita à terapêutica da HTA. A idade média foi de $54 \pm 4,3$ anos, o número médio de anos desde a licenciatura foi de $29 \pm 4,6$ anos, e $61,8 \%$ pertenciam ao sexo feminino. Comparando-se as variáveis dos médicos participantes no estudo e dos médicos que compõem a Rede de MS, verificou-se não existir diferenças significativas, para média de idade $(p=0,6737)$, para o número médio de anos de prática clínica $(p=0,5058)$ e para o sexo $(p=0,4023)$. É de salientar a diminuição do número de médicos participantes no estudo em $2010(\mathrm{n}=35)$. Decorrente deste facto, quando se compararam os doentes em estudo com a amostra de doentes que se esperava obter, verificou-se que, no ano de 1998, o número de médicos participantes foi superior ao esperado, re- sultando num tamanho da amostra superior ao previamente calculado. Em 2010, o número reduzido de médicos participantes resultou numa redução do tamanho amostral, que contou com aproximadamente metade dos participantes esperados.

\section{DISCUSSÃO}

As normas de orientação clínica para o tratamento da HTA da Joint National Committee 7, da European Society of Hypertension e European Society of Cardiology consideram que, de uma forma geral, independentemente do aHT utilizado, a monoterapia permite atingir o controlo da PA apenas num número limitado de hipertensos, sendo que a maioria dos doentes necessitará do uso de associações farmacológicas para atingir as metas tensionais desejáveis. ${ }^{9,10}$

No presente estudo, verificou-se que a utilização da combinação de vários aHT, isolados ou em associação de dose fixa, apresentou um aumento de 17,3\%, tendo sido prescrita a mais de metade dos hipertensos em 2010 (69,7\%). A proporção de doentes em monoterapia é inferior à observada no estudo VALSIM (2009), ${ }^{11}$ o que está de acordo com as observações de estudos noutros países europeus, que têm revelado que a proporção de doentes sob monoterapia tem vindo a diminuir signi- 
ficativamente, de forma constante. ${ }^{11}$

De acordo com as mais recentes normas de orientação clínica emanadas pela DGS, no tratamento da HTA de risco acrescido baixo, pode ser utilizado qualquer medicamento considerado de primeira linha - diuréticos tiazídicos, isolados ou em associação de baixa dose com modificadores do eixo renina angiotensina aldosterona (RAA); modificadores do eixo RAA (não diferenciando os IECA dos ARA) ou BCC. ${ }^{4}$ Contudo, determinadas condições de alto risco, como a diabetes mellitus, síndrome metabólica ou doença renal são indicações para o uso inicial de associações com mecanismos de acção complementar - diuréticos tiazídicos com modificadores do eixo RAA ou BCC com modificadores do eixo RAA. ${ }^{4}$

A escolha da opção terapêutica deve ser justificada sempre que se considere ser a melhor alternativa para o doente, seja para minimizar reacções adversas seja, eventualmente, para minorar o número de tomas; caso contrário, privilegia-se a opção terapêutica de menor custo para igual eficácia, salvaguardando o cumprimento das orientações de boa prática clínica. ${ }^{4}$

Neste estudo, embora os diuréticos tenham sido frequentemente prescritos em associação com outras classes de aHT (32,0\% em 1998 e 54,1\% em 2010), já quando prescritos em monoterapia representaram apenas $6,1 \%$ da utilização de aHT em 1998 e 2,2\% em 2010, apresentando uma diminuição de 3,9\% entre 1998 e 2010. Como fármacos de primeira linha, assim classificados pela maioria das normas de orientação clínica, ${ }^{4,10}$ e tendo em consideração que são a classe terapêutica com menor custo diário de tratamento, seria expectável uma maior proporção na sua utilização. ${ }^{12}$

Os fármacos que actuam no eixo RAA foram os mais frequentemente prescritos em ambos os momentos em estudo (1998 e 2010), tanto em monoterapia como em politerapia. Por outro lado, a utilização de diuréticos, BB e BCC diminuíram ou aumentaram residualmente entre os dois períodos.

Estes resultados estão de acordo com o observado nos últimos estudos nacionais sobre o padrão de utilização dos aHT, que revelaram que os IECA e, fundamentalmente, os ARA têm, cada vez mais, superado a utilização das outras classes de aHT, tais como os BCC e os BB. ${ }^{11,12} \mathrm{O}$ estudo VALSIM, publicado em 2009, demonstrou que os padrões de tratamento mais frequen- temente prescritos foram os ARA, em monoterapia $(16,9 \%)$, seguidos dos IECA em monoterapia $(14,4 \%)$ e das associações de ARA e diurético (11,8\%). ${ }^{11}$

Os ARA têm indicações semelhantes aos IECA, no entanto, devido ao seu elevado custo, devem ser apenas recomendados aos doentes que apresentem intolerância aos IECA., ${ }^{9,12,13,14}$ No presente estudo, o marcado aumento na utilização dos ARA e o decréscimo na proporção de utilização de IECA, parece sugerir a hipótese de que, em determinadas situações, estes poderão estar a ser utilizados para além das indicações em que representem uma mais-valia terapêutica, sugerindo-se a realização de outros estudos com outras opções metodológicas para a eventual comprovação desta hipótese. Não obstante, a atracção pela prescrição de moléculas novas, como é o caso dos ARA, e a pressão para usar as terapêuticas mais recentes são factores que podem afectar a prescrição do médico no tratamento da HTA, podendo explicar, parcialmente, a opção pelos ARA. ${ }^{13}$

Um estudo da Autoridade Nacional do Medicamento e Produtos de Saúde I.P. (INFARMED), que analisou a evolução dos medicamentos do aparelho cardiovascular em Portugal, entre 2000 e 2011, mostrou que os fármacos que actuam no eixo RAA apresentaram tendências de utilização crescentes, enquanto os restantes fármacos tiveram as suas tendências de utilização estabilizadas ou invertidas. ${ }^{15}$ Por outro lado, de acordo com os dados do Sistema de Informação sobre Consumo de Medicamentos do Centro de Estudos e Avaliação em Saúde (CEFAR), da Associação Nacional das Farmácias - sistema criado com base numa amostra de cerca de $80 \%$ das farmácias portuguesas, entre 2004 e 2011, a utilização dos aHT em Doses Diárias Definidas (DDD)/1.000 habitantes/dia aumentou 33,8\%. Neste período, o rácio em DDD: ARA/(IECA + ARA) - indicador do grupo EURO-MED-STAT ${ }^{16}$ - aumentou de 33,8\% para 51,9\%, evidenciando, claramente, a substituição dos IECA pelos ARA. De notar que desde 2005, e mais acentuadamente a partir de Outubro de 2010, o crescimento na despesa, apesar de elevado, foi bastante inferior ao crescimento em volume, devido às medidas legislativas implementadas em Portugal para redução dos custos, nomeadamente, reduções administrativas nos preços de venda ao público, quer dos medicamentos de marca, quer dos medicamentos genéricos. 
A HTA está frequentemente associada a determinadas patologias, com elevada prevalência, como a diabetes e a dislipidemia. Os doentes com HTA e determinadas comorbilidades necessitam de uma abordagem específica nas opções terapêuticas.

No caso de dislipidemia, as normas de orientação terapêutica desaconselham o uso dos $\mathrm{BB}$ em hipertensos com múltiplos factores de risco metabólico, incluindo a síndrome metabólica, devido aos seus efeitos adversos sobre o metabolismo lipídico e aumento da incidência da diabetes. ${ }^{10,17}$ Isto também se aplica ao uso de diuréticos tiazídicos, que em doses elevadas têm efeitos dislipidémicos e diabetogénicos. ${ }^{10} \mathrm{Em} 1998$, foi prescrito um IECA a $63,4 \%$ dos doentes com dislipidemia, enquanto em 2010 esta proporção diminuiu para $35,4 \%$, mantendo-se, contudo, como a classe mais frequentemente prescrita nos doentes cuja única comorbilidade era a dislipidemia.

Nos doentes com diabetes, o controlo da HTA pode ser particularmente difícil de alcançar, podendo ser necessária a utilização de uma combinação de dois ou mais aHT. ${ }^{10}$ É recomendado o uso de associações que incluam um IECA ou ARA, em virtude da eficácia particular destes agentes sobre a excreção renal de proteína e a preservação da função renal a longo prazo. ${ }^{14}$

A combinação de um diurético com um BB tem efeitos metabólicos adversos e favorece o desenvolvimento de diabetes, devendo, portanto, ser evitado, a menos que exigido por outras razões. Neste estudo, verificou-se que a proporção de hipertensos com diabetes aumentou significativamente de 1998 para 2010, pelo que poderá ter contribuído para o aumento da utilização de medicamentos modificadores do eixo RAA, os mais adequados para o tratamento da HTA em indivíduos diabéticos com proteinúria. ${ }^{10}$ Os fármacos mais frequentemente prescritos nos indivíduos cuja única comorbilidade era a diabetes foram os IECA, em 1998 (75\%), e a associação de IECA com diuréticos tiazídicos, em 2010 (52,9\%).

Os resultados deste estudo devem ser interpretados à luz de algumas limitações existentes. A generalização dos resultados deverá ser cuidadosa, uma vez que o número de hipertensos incluídos no estudo foi inferior ao tamanho amostral definido na metodologia e os padrões terapêuticos encontrados poderão não corresponder, na totalidade, aos padrões terapêuticos da ge- neralidade dos médicos de medicina geral e familiar em Portugal. Com efeito, o facto de os prescritores pertencerem à Rede de MS - rede voluntária de médicos de Medicina Geral e Familiar - constitui, por si só, um viés, na medida em que poderão apresentar um padrão de prescrição sistematicamente diferente do praticado por outros médicos de Medicina Geral e Familiar ou clínicos de outras especialidades, porventura reflexo da possibilidade dos utentes da Rede terem características diferentes e, no caso concreto, não serem representativos da população total de doentes a fazer terapêutica anti-hipertensora.

Embora o recrutamento dos doentes tenha sido efectuado sequencialmente, com base num plano de amostragem por cotas, previamente disponibilizado aos MS, é possível que tenha existido um viés de selecção, em virtude de uma selecção de conveniência dos doentes por parte dos MS e tendo em conta o longo período de recrutamento verificado no terreno.

Por outro lado, o facto de os médicos terem conhecimento de que se encontravam a participar num estudo com vista à caracterização do perfil de prescrição pode ter contribuído para um viés de notificação, considerando que cada médico participante pode não ter reportado algumas prescrições de aHT durante o período do estudo ou pode ter alterado o seu padrão habitual de prescrição.

Os períodos de recolha transversal de informação não possibilitam a obtenção de determinadas variáveis que poderiam permitir outras análises de relevância para a caracterização do perfil terapêutico da HTA, embora não constassem nos objectivos deste estudo. Não foi, por exemplo, recolhida informação sobre a experiência terapêutica anterior com aHT, como intolerância, efeitos adversos ou patologias associadas que contra-indiquem a utilização de algum aHT, bem como informação sobre terapêuticas concomitantes dos doentes, que permitiria a detecção de eventuais interacções medicamentosas. ${ }^{8}$

A forma de HTA avaliada neste estudo é referente à forma de HTA no momento do diagnóstico e a terapêutica é referente ao momento do estudo, em 2010. Entre estas duas datas podem mediar períodos de tempo variados, de anos até, pelo que não se pode estabelecer uma relação directa ente a terapêutica actual e a forma de HTA registada pelo médico. 
Em conclusão, verificou-se que o padrão de prescrição de aHT na Rede MS sofreu significativas alterações no que diz respeito ao número e tipologia de classes de aHT prescritos. O número médio de aHT prescritos aumentou significativamente entre os dois momentos. Por outro lado, embora os diuréticos sejam o aHT de menor custo, a sua utilização reduziu-se consideravelmente entre 1998 e 2010. Ao invés, os fármacos que actuam no eixo RAA (IECA e ARA) foram os mais frequentemente prescritos em ambos os momentos, tanto em monoterapia como em politerapia. Contudo, sublinha-se o aumento significativo da prescrição de ARA em detrimento da diminuição dos IECA.

Comparativamente ao primeiro estudo, salienta-se um ligeiro aumento da idade média dos participantes, mantendo-se porém o predomínio do sexo feminino. Aproximadamente metade dos hipertensos apresentou danos em órgãos-alvos em ambos os momentos, sendo a doença cardíaca o dano mais frequente. As patologias associadas mais frequentes foram a dislipidemia, a hiperuricemia e a diabetes. Verificou-se ainda uma variação significativa no número e classes de aHT prescritos em função da presença de comorbilidades nos dois momentos.

No âmbito do uso racional do medicamento, os resultados deste estudo contribuíram para o conhecimento do perfil de prescrição dos aHT em Portugal. Contudo, propõe-se, em estudos futuros, avaliar a implementação da Norma da DGS e o seu decorrente impacto no padrão de prescrição dos anti-hipertensores, por forma a promover a qualidade da prescrição e o real controlo da despesa pública com medicamentos, concretamente nesta classe farmacoterapêutica.

\section{AGRADECIMENTOS}

Um agradecimento especial a todos os Médicos Sentinela que tornaram possível este estudo enviando a informação necessária sobre os seus doentes e à Direcção da Associação Nacional das Farmácias pelo apoio concedido para a realização do estudo.

Ao Doutor Baltazar Nunes e à Dr ${ }^{\text {a }}$ Suzete Costa pela revisão do manuscrito e contributo para a discussão sobre a análise dos resultados.

\section{REFERÊNCIAS BIBLIOGRÁFICAS}

1. Morgado MP, Rolo SA, Pereira L, Castelo-Branco M. Blood pressure control and antihypertensive pharmacotherapy patterns in a hypertensive population of Eastern Central Region of Portugal. BMC Health Serv Res 2010 Dec 30; 10: 349.
2. De Macedo ME, Lima MJ, Silva AO, Alcântara P, Ramalhinho V, Carmona J. Prevalence, awareness, treatment and control of hypertension in Portugal: the PAP study. Rev Port Cardiol 2007 Jan; 26 (1): 21-39.

3. Perk J, De Backer G, Gohlke H, Graham I, Reiner Z, Verschuren M, et al. European Guidelines on cardiovascular disease prevention in clinical practice (version 2012). The Fifth Joint Task Force of the European Society of Cardiology and Other Societies on Cardiovascular Disease Prevention in Clinical Practice. Eur Heart J 2012 Jul; 33 (13): 1635-701.

4. Normas para a Abordagem Terapêutica da Hipertensão Arterial (Norma nº 026/2011 de 29/09/2011 actualizada a 19/03/2013). Lisboa: Direção-Geral da Saúde; 2011.

5. Polónia J, Ramalhinho V, Martins L, Saavedra J. Normas sobre detecção, avaliação e tratamento da hipertensão arterial da Sociedade Portuguesa de Hipertensão. Rev Port Cardiol 2006 Jun; 25 (6): 649-60.

6. Kithas PA, Supiano MA. Practical recommendations for treatment of hypertension in older patients. Vasc Health Risk Manag 2010 Aug 9; 6; 561-9.

7. Direcção de Serviços de Informação e Análise. Divisão de Estatística. Elementos Estatísticos: Informação Geral: Saúde 2008. Lisboa: DirecçãoGeral da Saúde; 2010.

8. Martins AP, Melo MN, Mendes Z, Ferreira AP, Miranda AC, Falcão IM, et al. Perfil terapêutico da hipertensão arterial na Rede dos Médicos Sentinela. Rev Port Clin Geral 2001 Set-Out; 17 (5): 359-72.

9. Chobanian AV, Bakris GL, Black HR, Cushman WC, Green LA, Izzo JL Jr, et al. The Seventh Report of the Joint National Committee on Prevention, Detection, Evaluation, and Treatment of High Blood Pressure: the JNC 7 report. JAMA 2003 May 21; 289 (19): 2560-72.

10. Mancia G, De Backer G, Dominiczak A, Cifkova R, Fagard R, Germano G, et al. 2007 ESH-ESC Practice Guidelines for the Management of Arterial Hypertension: ESH-ESC Task Force on the Management of Arterial Hypertension. J Hypertens 2007 Sep.; 25 (9): 1751-62.

11. Cortez-Dias N, Martins S, Belo A, Fiuza M. Prevalência e padrões de tratamento da hipertensão arterial nos cuidados de saúde primários em Portugal; resultados do Estudo VALSIM. Rev Port Cardiol 2009 Mai; 28 (5): 499-523.

12. Análise da evolução da utilização dos anti-hipertensores em Portugal Continental entre 1999 e 2003. Lisboa: Observatório do Medicamento e Produtos de Saúde - INFARMED; 2005.

13. Stafford RS, Monti V, Furberg CD, Ma J. Long-term and short-term changes in antihypertensive prescribing by office-based physicians in the United States. Hypertension 2006 Aug; 48 (2): 213-8.

14. Mancia G, Laurent S, Agabiti-Rosei E,Ambrosioni E, Burnier M, Caulfield MJ, et al. Reappraisal of European guidelines on hypertension management: a European Society of Hypertension Task Force document. Blood Press 2009; 18 (6): 308-47.

15. Medicamentos do Aparelho Cardiovascular: Uma análise dos padrões de utilização e despesa em Portugal Continental entre 2000 e 2011. Lisboa: Infarmed; 2012.

16. STAT, EURO MED. The Library of European Union Pharmaceutical Indicators Expenditure and Utilisation Indicators. Final version. March 2004. Disponível em http://ec.europa.eu/health/ph_projects/2001/monitoring/fp_monitoring_2001_frep_12_3_en.pdf [acedido em 25/09/2013].

17. Bangalore S, Parkar S, Grossman E, Messerli FH. A meta-analysis of 94,492 patients with hypertension treated with beta blockers to de- 
termine the risk of new-onset diabetes mellitus. Am J Cardiol 2007 Oct 15; 100 (8): 1254-62.

\section{CONFLITO DE INTERESSES}

O autor José Augusto Simões declara que nos últimos três anos foi prelector em eventos científicos patrocinados pela Merck, Sharp and Dhome, pela Novartis e pela Pfizer e foi também perito externo na avaliação das Normas de Orientação Clínica da Direcção Geral da Saúde. Os restantes autores declaram não possuir qualquer conflito de interesses.

\author{
ENDEREÇO PARA CORRESPONDÊNCIA \\ Carla Torre \\ CEFAR-Centro de Estudos e Avaliação em Saúde \\ Associação Nacional das Farmácias \\ Rua Marechal Saldanha, 1 \\ 1249-069 Lisboa \\ Email: carla.torre@anf.pt
}

Recebido em 25-04-2013

Aceite para publicação em 15-10-2013

\section{ABSTRACT}

\section{PRESCRIBING PATTERNS FOR HYPERTENSION IN THE PORTUGUESE SENTINEL PRACTICE NETWORK} -12 YEARS AFTER

Objectives: To determine the treatment profile of hypertension in the Portuguese sentinel practice network and compare the results 12 years after an initial study.

Study design: Cross-sectional study

Setting: Portuguese sentinel practice network

Participants: Hypertensive patients over 18 years of age on the lists of general practitioners in the sentinel practice network who attended a consultation with their physician between June 7, 2010 and December 31, 2010.

Results: In 2010, 616 hypertensive patients were included in the study. Between the two periods (1998 and 2010) there was a slight increase in the average age of the participants (62.0 years in 1998 and 64.3 years in 2010), with a higher proportion of female patients in both periods. The mean average duration of hypertension since the time of diagnosis increased significantly $(<0.0001)$. Approximately half of the hypertensive patients presented target organ damage, in both periods with heart disease as the most common lesion. Dyslipidemia, hyperuricemia and diabetes were the most common co-morbid conditions. From 1998 to 2010 , the proportion of patients on monotherapy decreased from $47.6 \%$ to $30.3 \%$. Angiotensin-converting enzyme inhibitors were the most frequently prescribed drugs for monotherapy in both periods. In 1998, the antihypertensive treatment most frequently prescribed was angiotensin-converting enzyme inhibitors as monotherapy (24.3\%). In 2010 the combination of angiotensin receptor blockers with thiazide diuretics was the most common treatment (15.4\%).

Conclusions: Drugs acting on the renin-angiotensin system were the antihypertensive drugs most frequently prescribed in both periods as monotherapy and in combination with other drugs. The number of antihypertensive drugs prescribed differed between the two periods in relation to the existence and type of co-morbidities.

Keywords: Hypertension; Physician Prescribing Pattern; Therapeutic Uses; Primary Care Physicians. 